\title{
Dendritic End Inhibition in Large-Field Visual Neurons of the Fly
}

\author{
Yishai M. Elyada, Juergen Haag, and Alexander Borst \\ Max Planck Institute of Neurobiology, 82152 Martinsried, Germany
}

The extraction of optic flow fields by visual systems is crucial for course stabilization during locomotion, and relies on feedforward and lateral integration of visual inputs. Here we report a novel form of systemic, motion-sensitive lateral suppression in the dendrites of large, flow-field-selective neurons in the fly's visual lobes. Using in vivo Calcium-imaging and intracellular recordings, we demonstrate that responses in dendrites, but not axon terminals, are end inhibited by flanking gratings both in the vertical and horizontal systems. We show evidence for a mechanism involving wide-field dendritic inhibition that exceeds the retinotopic spatial extent of the dendrites. Using compartmental modeling, we point out a possible function in enhancing selectivity for optic flow fields. Our results suggest that lateral suppression is a common element serving similar functional requirements in different visual systems.

\section{Introduction}

In many organisms, fast visual motor systems direct gaze and course stabilization behaviors that stabilize retinal images and ensure proper locomotion. The visual motion patterns that are relevant for these behaviors are complex, large flow-field patterns that arise from different spatial maneuvers, and populations of cells that respond to such flow fields have been found in various species (Tanaka et al., 1989; Duffy and Wurtz, 1991; Toyama et al., 1994; Krapp and Hengstenberg, 1996; Wylie et al., 1998; Britten, 2008; Borst et al., 2010). The mechanisms that underlie the selectivity of neurons to such spatiotemporal patterns are generally understood to comprise two classes of operations-selective integration of retinotopic feedforward inputs (Tanaka et al., 1989; Duffy and Wurtz, 1991) and horizontal interactions between flow-field-selective neurons (Krapp et al., 2001; Haag and Borst, 2004; Farrow et al., 2006).

The visual system of the blowfly, Calliphora vicina, is a well characterized model for optic flow processing in which these mechanisms are known in extensive detail (Hausen and Egelhaaf, 1989; Egelhaaf and Warzecha, 1999; Borst et al., 2010; Borst and Weber, 2011). In this system, retinotopic visual inputs from local motion detectors (LMDs) are integrated by large-field lobula plate tangential cells (LPTCs) (Fig. 1a). By integrating these feedforward signals (Borst and Egelhaaf, 1992; Single and Borst, 1998; Borst and Single, 2000), and through lateral interactions within

\footnotetext{
Received Aug. 28, 2012; revised Dec. 27, 2012; accepted Jan. 10, 2013.

Author contributions: Y.M.E., J.H., and A.B. designed research; Y.M.E. performed research; Y.M.E. analyzed data; Y.M.E., J.H., and A.B. wrote the paper.

This work was supported by the Max Planck Society and the Bernstein Center for Computational Neuroscience Munich. Y.M.E. was supported partially by a grant from the Human Frontiers Science Program (Max Planck Florida Institute). We thank M. Huebener for comments on an earlier version of this work; V. Bhandawhat for helpful discussions; and R. Gleich for technical assistance.

The authors declare no competing financial interests.

Correspondence should be addressed to Yishai M. Elyada, Max Planck Florida Institute, 1 Max Planck Way, Jupiter, FL 33458. E-mail: yishai.elyada@mpfi.org.

DOI:10.1523/JNEUROSCI.4136-12.2013

Copyright $\odot 2013$ the authors $\quad 0270-6474 / 13 / 333659-10 \$ 15.00 / 0$
}

the bilateral lobula plate system (Farrow et al., 2003, 2006; Haag and Borst, 2003, 2007; Borst et al., 2010), LPTCs acquire complex receptive fields (RFs) that tune them to optic flow-field patterns induced by different flight maneuvers (Krapp and Hengstenberg, 1996; Franz and Krapp, 2000; Krapp et al., 2001). This representation of the optic flow field, output at the axon terminal, is used by downstream neurons to control the fly's motor behavior (Heisenberg et al., 1978; Geiger and Nässel, 1981; Hausen and Wehrhahn, 1990; Haag et al., 2010).

The LMD inputs are a mixture of motion-opponent excitatory and inhibitory subunits, with the direction of motion determining their balance and the resulting postsynaptic response (Borst et al., 1995). It has been long thought that the excitatory and inhibitory components of the LMDs innervate the LPTCs in a strictly feedforward fashion where the LMDs are tuned to small, non-overlapping domains in visual space. In each LMD, the opponent subunits are thought to be spatially congruent.

Using in vivo intracellular recordings and imaging of calcium responses (Borst and Egelhaaf, 1992; Egelhaaf and Borst, 1995; Haag and Borst, 2000), we show that the responses in the dendrites of LPTCs are suppressed by large-field stimuli that extend beyond the spatial integration profiles of their dendrites, rendering them selective for small-field motion. We show that this suppression extends only in the direction perpendicular to the preferred direction of the cells. We show evidence for a mechanism involving lateral inhibition (LI) with a spatial profile that is broader than the concurrent excitatory inputs. Using a compartmental model, we suggest a function in enhancing selectivity to rotational flow fields in LPTCs of the vertical system (VS) cells.

\section{Materials and Methods}

Electrophysiology. Experiments were carried out as described previously (Elyada et al., 2009). Briefly, female blowflies (2-4 d old, laboratory stock) were anesthetized under $\mathrm{CO}_{2}$, immobilized with wax and an opening was made in the dorsal cuticle. Trachea and air sacs were removed, exposing the lobula plate. VS cells were recorded from the dendritic branching point using micropipettes with a resistance of $\sim 20 \mathrm{M} \Omega$, con- 
a

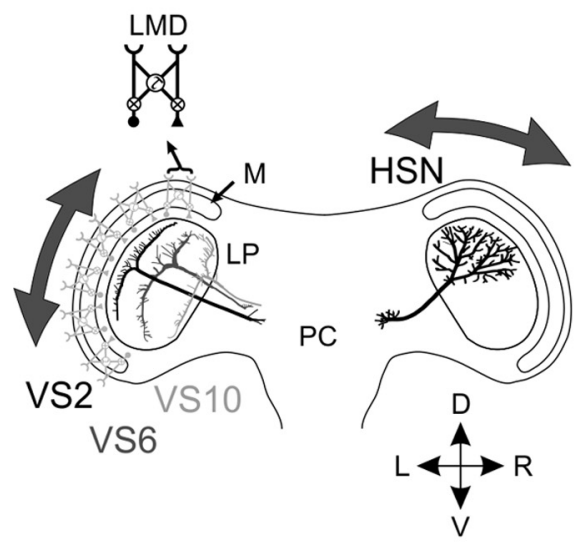

b 중

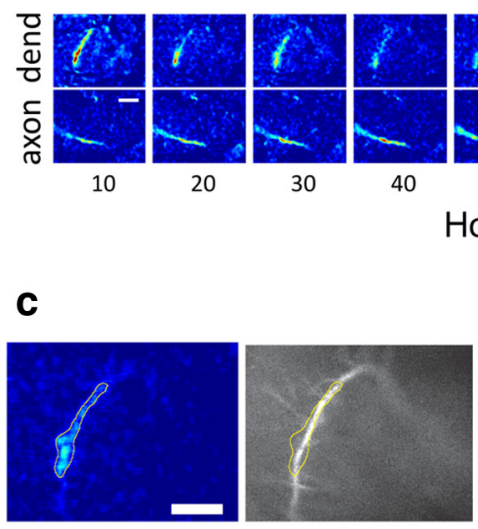

E三

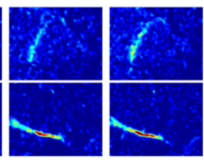

50

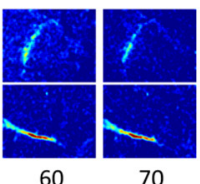

60

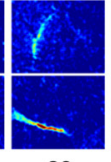

80

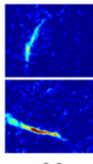

90
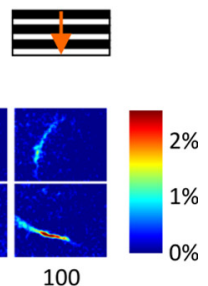

100
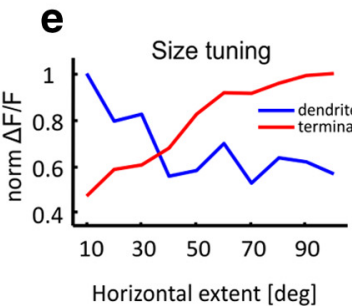

f

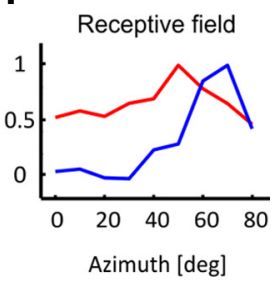

Figure 1. End inhibition in a VS cell. $\boldsymbol{a}$, Schematic of the fly visual system, seen from the back of the head. An array of Reichardt-type LMDs presynaptic to the lobula plate calculate the multiplication (circled X) of two luminance signals from adjacent photoreceptors (half circles) after low-pass filtering of one of them $(\tau)$, resulting in excitation for one direction (triangle) and inhibition for the other (circle). The left hemisphere shows 3 of 10 VS cells, and the right hemisphere shows 1 of 3 HS cells. LP, Lobula plate; M, medulla; PC, protocerebrum; D, dorsal; V, ventral; L, left; $R$, right. $\boldsymbol{b}$, Calcium responses ( $\triangle F / F)$ in the dendrite and axon terminal of a VS4 cell to gratings of varying horizontal extent drifting in the preferred direction of the cell. $c$, Definition of the ROI for this cell, based on its calcium response to the $100^{\circ}$ stimulus. $\boldsymbol{d}$, Same ROI plotted on a raw imaging data frame. $\boldsymbol{e}$, Normalized, ROl-averaged calcium responses as a function of the horizontal extent of a grating drifting downwards. $f$, Dendritic and axon terminal calcium responses as a function of the azimuthal position of a $20^{\circ}$ wide horizontal grating drifting downward. Scale bars, $100 \mu \mathrm{m}$.

taining OGB-1 and Alexa Fluor 594 for Calcium-imaging, or Alexa Fluor 594 (Invitrogen) for current injection (discontinuous mode, switching frequency $20-40 \mathrm{kHz}$ ). Drugs were dissolved in the same tip solutions. We carefully compensated electrode capacitance by balancing out an injected $200 \mathrm{~Hz}$ sinusoidal current that is slow enough to drive a capacitive response in the electrode, but not in the cells (corner frequency 80 $\mathrm{Hz}^{9}$ ). Electrode capacitance was reduced by coating the pipette shaft with wax.

Visual stimulation. Visual stimuli for the VS cell imaging experiments (Figs. 1, 2) were presented on a $300 \mathrm{~Hz}$ CRT monitor (Image Systems; maximum luminance, $97.7 \mathrm{~cd} \mathrm{~m}^{-2}$; pixel pitch, $0.29 \mathrm{~mm}$; field of view, $108^{\circ}$ ) in pseudorandom order. For dendritic and axon terminal receptive-field mapping, we presented $20^{\circ}$ wide square wave gratings $\left(20^{\circ}\right.$ spatial period, $46^{\circ}$ height, $20^{\circ}$ width) drifting downward with a $3 \mathrm{~Hz}$ temporal frequency at lateral azimuths from $0^{\circ}$ (frontal) to $80^{\circ}$ (lateral). In the size-dependence experiments, we used a $10^{\circ}$ "center" grating at the peak of the dendritic RF and increased its size by $10^{\circ}$ increments in both directions until the edge of the screen was reached; further increments were obtained only in the remaining direction. Stimuli were presented for $3 \mathrm{~s}$ in the calcium-imaging experiments (interstimulus interval $8 \mathrm{~s}$ ), and twice for $2 \mathrm{~s}$ separated by $2 \mathrm{~s}$ without motion in the impedance measurement experiments (interstimulus interval, $5 \mathrm{~s}$ ).

For the horizontal system (HS) cell imaging experiments (Figs. 3, 4) and for the current injection experiments (Figs. 5, 6), we used a $75 \mathrm{~Hz}$ TFT monitor (Dell; E151FPb; maximum luminance, $212 \mathrm{~cd} \mathrm{~m}^{-2}$; pixel pitch, $0.297 \mathrm{~mm}$; response time, $30 \mathrm{~ms}$ ). Sine wave gratings were used to avoid phase locking of the visual responses to the refresh rate of the screen during motion. In the HS experiments, visual stimuli were adapted from the vertical to the horizontal dimension, using gratings drifting front-to-back in the HS cells' preferred direction.

Calcium imaging. Calcium imaging was performed using an epifluorescence CCD camera at $2 \mathrm{~Hz}$ (CoolSnapHQ, Roper Scientific) using a $20 \times$ water-immersion objective (UMPLFLN 20XW, Olympus) and a mercury arc lamp (HBO100, OSRAM). Calcium-imaging stacks were corrected for motion artifacts (ImageJ, stackreg extension), and $\Delta F / F$ stacks were filtered with a $10 \times 10$ pixel median filter and pixel-wise corrected for bleaching by subtracting an exponential fit over time for the first three frames and last frame.

To quantify the calcium responses, we calculated difference images between the averages of three frames during the stimulus and three be- fore stimulus onset, then defined the region of interest (ROI) using a $100^{\circ}$ (VS cells) or $10^{\circ}$ (HS cells) grating by defining a threshold set as $30 \%$ of the value of the 95th percentile pixel, and averaged the $\Delta F / F$ value over all pixels in the ROI.

Modeling. For our simulations, we used a previously published model (Elyada et al., 2009), consisting of a 20-compartment network with a dendritic and an axon terminal compartment for each cell, connected by an axonal conductance. Gap junctions between the axon terminals were simulated by conductances. The parameters for the models were fitted to current injection responses in an anatomically realistic model (Cuntz et al., 2007). We included only passive conductances in the dendritic and axon terminal compartments for simplicity. Image motion movies representing the ipsilateral visual field $\left(0-180^{\circ}\right.$ azimuth) were passed through an array of Reichardt-type LMDs. For input, we used a $100 \times$ 100 pixel checkerboard image with $5 \times 5$ pixel tiles filtered by a Gaussian with $\sigma=2$ pixels that was rotated at $50^{\circ} \%$ s or translated at $59.5877^{\circ} \%$ s to match the downward rotation speed at the edge of the image (i.e., the tangential speed of the rotation). The outputs of the LMDs were scaled and fed into the dendritic compartments as inhibitory and excitatory conductances (for more details, see Elyada et al., 2009). To simulate lateral inhibition, for each model we chose a certain proportion of the total inhibitory conductances (20-80\%, "percentage lateral inhibition") and replaced it by lateral inhibition inputs. These inputs were constructed by taking the excitatory inputs over a certain extent of lateral inhibition (a choice between $36^{\circ}$ and $180^{\circ}$ for each model), multiplying it by the proportion of inhibitory inputs removed, and feeding it into the dendritic compartment of the cell.

\section{Results}

We presented flies with gratings drifting in the preferred direction of VS cells, while imaging dendritic and axon terminal calcium influx using the calcium sensitive dye OGB-1. In VS cells, dendritic calcium responses are thought to reflect local, retinotopic LMD input (Single and Borst, 1998), whereas axon terminal responses include a component propagated from the dendrite as well as lateral, gap-junction inputs from neighboring VS cells (Elyada et al., 2009). First, we mapped the RFs of the dendrites and axon terminals using $20^{\circ}$ gratings. We then presented gratings of varying horizontal extents around the center of the den- 
a

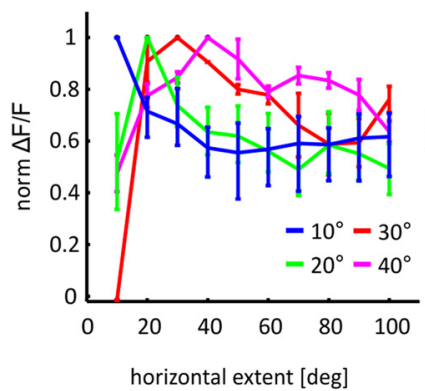

b

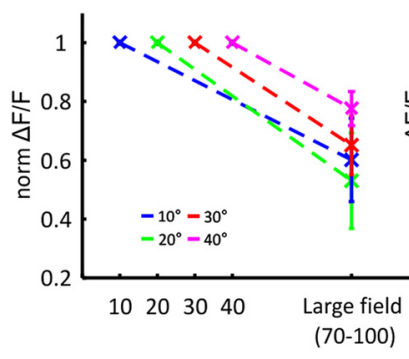

horizontal extent [deg]
C

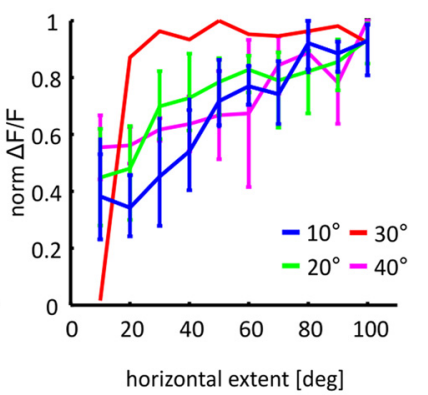

d

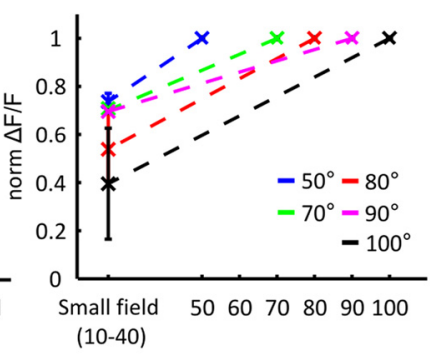

horizontal extent [deg]

Figure 2. Size dependence of responses in dendrites and axon terminals of VS cells. $\boldsymbol{a}$, Normalized dendritic calcium responses to gratings of varying horizontal extent, grouped according to the maximum dendritic response. $\boldsymbol{b}$, Comparison of the average dendritic responses for large-field stimuli $\left(70-100^{\circ}\right)$ versus peak responses. c, Same as $\boldsymbol{a}$ for axon terminal calcium responses, using the same grouping. $\boldsymbol{d}$, Comparison of the average axon terminal responses for small-field stimuli $\left(10-40^{\circ}\right)$ versus peak responses, grouped according to the maximum axon terminal response.

dritic RF. When we increased the horizontal extent of the gratings from $10^{\circ}$ to $100^{\circ}$, calcium responses in the axon terminals increased with the horizontal extent of the stimulus, as demonstrated in an example VS4 cell (Fig. 1b). This is expected because the axon terminals of VS cells integrate responses from neighboring VS cells, whose RFs cover adjacent parts of visual space (Elyada et al., 2009). However, in the dendrite, the strongest response was to a small-field stimulus, in this case of only $10^{\circ}$ horizontal extent. This result is surprising because we expected feedforward, retinotopic integration of input, and we did not expect suppression from outside the dendritic RF. We refer to this phenomenon as "end inhibition."

We quantified our finding by defining an ROI in the dendrite (Fig. 1c,d) and the axon terminal (data not shown) as all pixels that respond to a full-field stimulus. ROI averaged responses from Figure $1 b$ are shown in Figure $1, e$ and $f$; in the axon terminal, the calcium response to the largest stimulus was twice as large as to the smallest stimulus, whereas in the dendrite, the response dropped sharply from its peak at $10^{\circ}$ to nearly half $(55.3 \%)$ at $40^{\circ}$.

We analyzed calcium responses as well as intracellular membrane potential responses in 19 VS cells (4 VS2, 7 VS3, 7 VS4, 1 VS5). In receptive field mapping experiments, membrane potential responses recorded in this location correspond well to calcium responses in the axon terminal (Elyada et al., 2009). In the dendrites, 15 cells had peak calcium responses at $10-20^{\circ}, 2$ cells at $30^{\circ}$, and another 2 cells at $40^{\circ}$ (Fig. $2 a$ ). In all dendrites, the average response to large-field gratings $\left(70-100^{\circ}\right)$ was significantly smaller than to the strongest stimulus (Fig. $2 b ; p<10^{-12}$, Wilcoxon sign rank test), showing average reductions of between $22 \%$ and $47 \%$. In all cells, the strongest axon terminal calcium response we obtained was to gratings of $\geq 50^{\circ}$ (Fig. $2 c, d$ ), and was

a

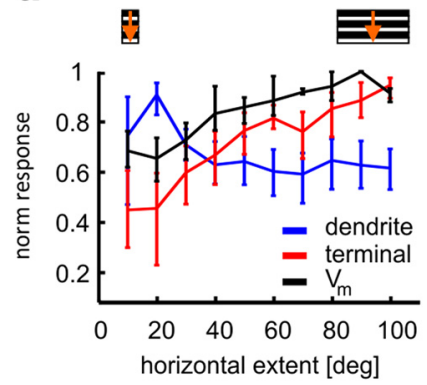

b

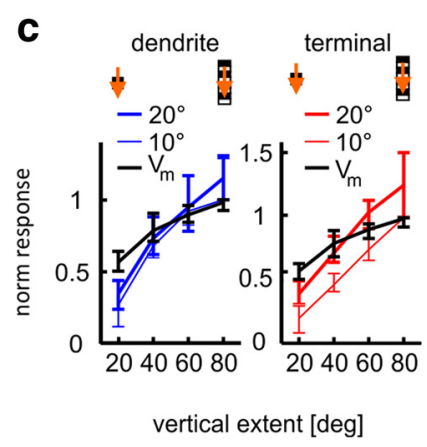

d

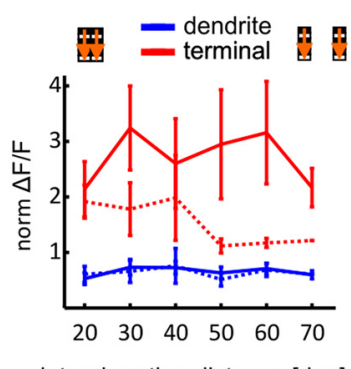

lateral grating distance [deg]

Figure 3. End inhibition in VS cells - population analysis. $\boldsymbol{a}$, Membrane potential responses (black) and calcium responses in the dendrites (blue) and axon terminals (red) of VS cells to gratings of varying horizontal extent drifting in the preferred direction (means with $95 \%$ bootstrap confidence intervals, $n=13$ cells). Normalized membrane potential for $V_{\mathrm{m}}$ traces, normalized $\Delta F / F$ for calcium-imaging traces for all panels. $\boldsymbol{b}$, Calcium responses in dendrites and axon terminals as in $\boldsymbol{a}$, compared with the arithmetic summation prediction of dendritic responses constructed from the receptive field. $c$, Responses to gratings of varying vertical extent drifting in the preferred direction. Gratings were either $10^{\circ}$ or $20^{\circ}$ wide, responses from each cell normalized to longest $10^{\circ}$ grating. $\boldsymbol{d}$, The effect of adding a laterally positioned $20^{\circ}$ grating on the calcium responses to a grating in the center of the dendritic receptive field ( $n=10$ cells). Solid lines are lateral displacements toward the center of the visual field, broken lines toward the periphery. $\Delta F / F$ responses normalized to responses to central grating (see Results).

significantly larger than the responses to small-field gratings (10$40^{\circ}$ horizontal extent, $p<10^{-10}$, Wilcoxon sign rank test).

\section{Spatial profile of VS cell suppression}

To allow for a unified stimulation paradigm that includes a test for lateral inhibition, we restricted the analysis to all VS cells in which dendritic peak calcium responses were at $10-20^{\circ}$, comprising $79 \%$ of the entire dataset. This allowed us to compare responses between one and two $20^{\circ}$ gratings without confounding increases and decreases in responses. In these cells, axon terminal responses grew steadily from $\sim 50 \%$ of the peak response at $10-$ $20^{\circ}$ to the peak response at $100^{\circ}$ (Fig. 3a). The graded potential responses of the membrane potential $\left(V_{\mathrm{m}}\right)$ grew from $70 \%$ at $10-20^{\circ}$ to a peak at $90^{\circ}$. In both cases, the increase continued well beyond the lateral extent of the dendritic receptive field $\left(\sim 30^{\circ}\right)$ (Elyada et al., 2009), and also beyond what would be expected from linear integration of the dendritic responses (Fig. 3b). Responses in the dendrites peaked at $10^{\circ}$ or $20^{\circ}$ and decreased to a constant level of $\sim 60 \%$ of the peak response at a horizontal extent of only $40^{\circ}$ (Fig. $3 a$ ), corresponding to the horizontal extent of the dendritic receptive field (Fig. 3b). Increasing the vertical extent of the stimulus resulted in a linear increase in calcium responses both in the dendrites and in the axon terminals (Fig. $3 c$ ). This shows that the decrease in dendritic calcium influx with horizontal stimulus size is specific to that dimension, and that it is not a result of different imaging conditions in the dendrites. 
We next asked whether the underlying mechanism corresponds to classical lateral inhibition, in which the suppression declines with distance from the receptive field center. For 10 VS cells, we presented two $20^{\circ}$ gratings simultaneously, one in the center of the dendritic RF (central grating) and another at varying horizontal displacements (lateral grating). In the dendrites, we saw no positional effect of the lateral grating on the response to the central grating (Fig. $3 d$ ). In the axon terminals, lateral gratings caused a relatively constant increase in the calcium response when they were centrally positioned, but this increase showed a reduction in magnitude with distance for lateral gratings placed toward the periphery.

\section{End inhibition in HS cells}

In the visual system of flies, motion in both the vertical and horizontal axes is represented by the responses of dedicated groups of VS and HS cells. We next asked whether end inhibition is specific to VS cells, or whether it is inherent in the fly motion detection pathway, and can therefore be found also in HS cells. We presented gratings drifting in the preferred direction of these cells while imaging calcium responses in the dendrites and axon terminals, modifying the stimulus paradigm to the horizontal dimension by a $90^{\circ}$ rotation. In an example HSE cell (Fig. 4), the axon terminal responses increased with the vertical extent of the stimulus, saturating at $30^{\circ}$ (Fig. $4 a$ ). In the dendrite, however, local responses showed a pronounced reduction in amplitude with growing vertical extent. To quantify the decrease in dendritic responses, and because HS cell dendrites are not confined to narrow, horizontal stripes of the lobula plate as the VS cells are to vertical stripes, we limited our ROI to small dendritic regions that were maximally activated by the smallest grating. This was done to avoid confounding the increased area of activation to the larger stimuli with decreased activation due to end inhibition (Fig. $4 b, c$ ). The resulting activation curve in the dendritic ROI decreased monotonically with increasing vertical extent down to $50 \%$ of the maximum response, in contrast to the saturating axon terminal response.

We performed similar analysis on 14 HS cells (6 HSN, 7 HSE, and 1 HSS). In all these cells, both calcium responses in the axon terminal and membrane potential responses increased with the vertical extent of the stimulus. In the dendrite, of the 14 cells, all but 3 cells responded maximally to stimuli of $30^{\circ}$ vertical extent or smaller. We normalized each cell's response curves to their maximum and plotted the average over all cells' dendrites, axon terminals, and membrane potentials in Figure $5 a$. The dendritic average normalized response curve dropped to $75 \%$ at vertical extents of $60-70^{\circ}$, and the responses to $60-80^{\circ}$ are significantly smaller than the responses to $10-30^{\circ}$ ( $p=0.0085$, Wilcoxon rank sum test). This decrease in responses with vertical stimulus extent is seen when the responses curves are grouped according to their strongest response, making each normalized curve peak at 1 (Figs. $5 b, c$ ). In all axon terminals, responses to $10-30^{\circ}$ were smaller (mean 31\%) than the peak response, which was to gratings $>40^{\circ}$ in all cases $(p=0.0313$, Wilcoxon rank sum test). We saw no decrease in dendritic responses with increase in horizontal extent (Fig. 5e); responses did not increase as we had observed in VS cell dendrites due to the small-field ROIs. We saw an increase in axon terminal and membrane potential responses with horizontal stimulus extent (Fig. $5 f$ ). These observations are expected from the integration of dendritic responses by the axon terminal.

\section{Mechanism of end inhibition in LPTCs}

To account for the suppression effects of laterally positioned gratings on the dendritic responses of LPTCs, we considered two possible mechanisms. Lateral inhibition could act directly on the dendrites of the LPTCs, or the suppression could act further upstream, causing an overall reduction in the excitatory LMD inputs onto the cells. In the first case, synaptic conductance is expected to increase with the opening of more inhibitory channels, and in the second case it is expected to decrease as less excitatory channels are opened. To distinguish between these two possibilities, we used current injections to measure the dependence of membrane impedance on the horizontal extent of the visual stimulus in VS cells.

To prevent the opening of strong voltage-gated potassium and sodium channels (Haag et al., 1997), recording micropipettes were filled with $100 \mathrm{~mm}$ tetraethylammonium (TEA) and $10 \mathrm{~mm}$ QX-314. This eliminated the rectifying voltage-gated currents that resulted from positive current injections (Fig. $6 a$ ), causing a linearization of the $I-V$ curve of these cells (Fig. $6 b$ ). To estimate changes in the cells' impedance, we injected current pulses before and during motion and recorded the resulting membrane potential deflections (Fig. $6 c$ ). Increasing the horizontal extent of the stimulus resulted in a decrease of membrane impedance (Fig. $6 d$ ), supporting the notion of direct dendritic inhibition. We repeated these experiments in seven VS cells, and the average impedance decreased with size of the stimulus from $10^{\circ}$ to $60^{\circ}$, after which is was constant at $\sim 77 \%$ of the impedance at the maximum $\left(10^{\circ}\right.$ stimulus; Fig. $\left.6 e\right)$.

\section{Enhancing selectivity for rotation}

We next asked, what is the function of end inhibition in the LPTC network? This question is particularly puzzling in the context of 

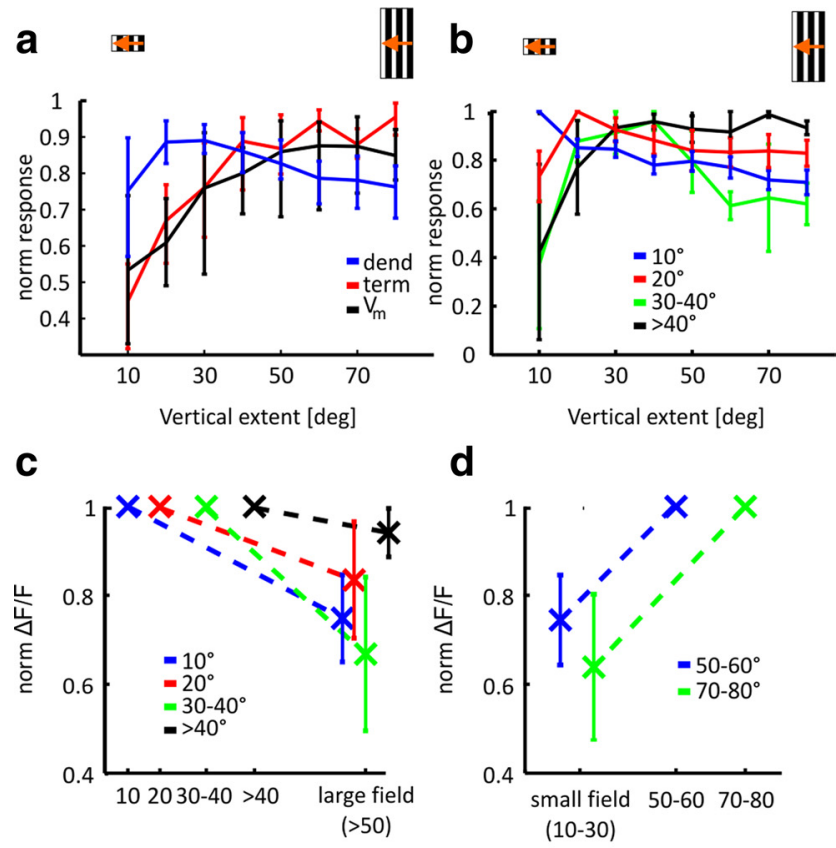

d

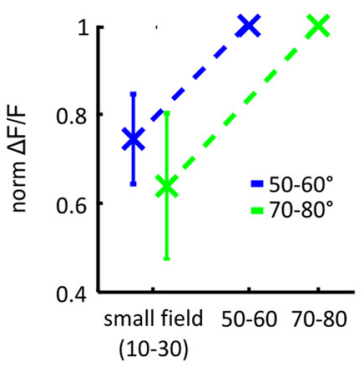

vertical extent $[\mathrm{deg}]$

vertical extent $[\mathrm{deg}]$

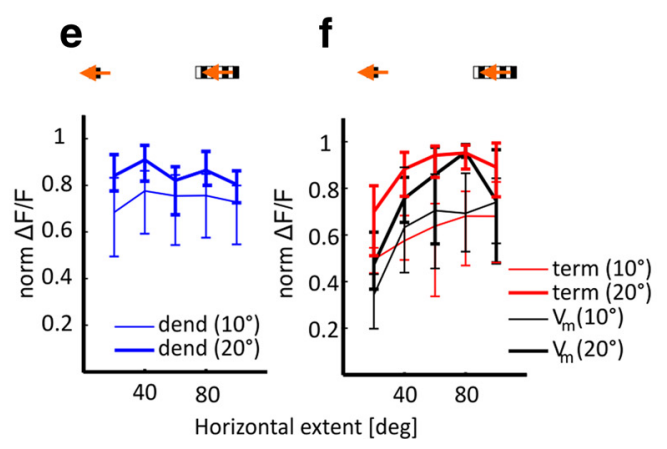

Figure 5. End inhibition in HS cells — population analysis. $\boldsymbol{a}$, Membrane potential responses (black) and calcium responses in the dendrites (blue) and axon terminals (red) of $\mathrm{HS}$ cells ( $n=$ 14,6 HSN, 7 HSE, 1 HSS) to gratings of varying vertical extent drifting in the preferred direction (means with $95 \%$ bootstrap confidence intervals). Normalized membrane potential for $V_{m}$ traces, normalized $\Delta F / F$ for calcium-imaging traces for all panels. $\boldsymbol{b}$, Same data as in $\boldsymbol{a}$, grouped according to vertical extent of the maximal response grating. $c$, Comparison of the average dendritic responses for large-field stimuli $\left(50-80^{\circ}\right)$ versus peak responses, grouped according to $\boldsymbol{b}$. $\boldsymbol{d}$, Comparison of the average axon terminal responses to small-field stimuli $\left(10-30^{\circ}\right)$ versus peak response, grouped according to maximal axon terminal response. $\boldsymbol{e}$, Dendritic responses to gratings of varying horizontal extent drifting in the preferred direction. Gratings were either $10^{\circ}$ or $20^{\circ}$ wide. $\boldsymbol{f}$, Same as $\boldsymbol{e}$ for axon terminal responses (red) and membrane potential (black).

VS cells, whose main output sites are in their axon terminals. The possibility for horizontal gap-junction connectivity in the dendrites of VS1 and VS7-10 was raised in a previous study (Haag and Borst 2007); however, the location of these outputs is yet to be confirmed anatomically. In the axon terminals, the small-field selectivity evident in the dendrites is completely abolished by lateral, gap-junction-mediated excitation from neighboring VS cells. Previous work showed that VS cells play an important role in the representation of visual flow fields resulting from rotation around the fly's own axis (Krapp and Hengstenberg, 1996; Franz and Krapp, 2000).

What is the contribution of lateral inhibition to this function of the LPTC network? Addressing this question experimentally is problematic, because systemic GABA blockade also blocks the
LMD inhibitory subunits (Egelhaaf et al., 1990; Borst et al., 1995). We therefore turned to a previously published compartmental model, which we constructed by reducing an anatomically precise model of a VS network to a model in which each cell has a dendritic and an axonal compartment (Cuntz et al., 2007; Elyada et al., 2009) (see Materials and Methods). In both these previous models, the inhibitory and excitatory LMD inputs were constructed using data from full-field motion stimulation. We reasoned that in an LI network, full-field stimulation would elicit the same inhibitory currents, but each VS cell will receive its inhibitory inputs from a broad spatial profile. We therefore compared the original model with strictly feedforward LMD inputs, with a set of 25 models in which inhibitory inputs incorporate a varying proportion of LI (Fig. 7). In each model, a different percentage of the total visually evoked inhibitory conductance $(20-100 \%)$ was replaced by LI inputs taken from a different lateral summation window $\left(36-180^{\circ}\right)$. The amplitudes of the LI inputs are proportional to the excitatory, preferred direction inputs in this summation window, reflecting the results we show in Figure 1. We reasoned that LI would be weaker for a visual input from an image rotating around the preferred axis of rotation of a cell (Fig. $7 a)$ than from a translating image drifting in the preferred direction (Fig. 7b), since in the former, significant parts of the visual field do not drift in the preferred direction and would thus give rise to less LI (Fig. 7, compare $c, d$ ). However, this prediction is not trivial because depolarizing currents from neighboring VS cells, mediated by gap-junction connections in the axon terminals, may act to negate the effects of LI.

We first tested in all models whether LI can result in a smallfield selective response in the dendrite, together with a large-field selective response in the axon terminal. This was the case in all models except for those with a LI extent of $36^{\circ}$ (results not shown), which are incompatible with the experimental results (Fig. 3d). In the case of our best-fitted model (Fig. 7e), dendritic responses to gratings $>50^{\circ}$ wide were reduced by $>50 \%$, but in the axon terminal the largest grating elicited a response fivefold stronger than the smallest.

In VS cells, selectivity to rotational flow fields (Wertz et al., 2009) is mediated mainly by horizontal motion inputs (Krapp and Hengstenberg, 1996; Franz and Krapp, 2000), which rely on complex network interactions with other LPTCs, mainly from the horizontally system (Borst and Weber, 2011). We focused our model on the contribution of vertical motion inputs to flow-field selectivity, and asked whether broadening the spatial profile of LI, even without horizontal inputs, can result in selective suppression of responses to translation. Responses to rotation around the center of the image in VS1's axon terminal were reduced 36\% from 19 to $12.2 \mathrm{mV}$ by the addition of LI (Fig. $7 f$ ), but the reduction was 53\% from 30.4 to $14.4 \mathrm{mV}$ during translation, and $60 \%$ in VS5, owing to edge effects in the LI model (Fig. $7 g$ ). Analogous results can be obtained for other axes of rotation because of the symmetrical structure of the VS network, around each cell's axis of rotation. We quantified this by calculating the ratio of suppression during translation to that during rotation for all models (Fig. $7 h)$. This ratio was larger than unity in all the models with LI extent $>36^{\circ}$, and ranged between 1.46 and 2 for the four models we consider as the ones most fitting our experimental data (Fig. 7i). Such ratios are indicative of increased selectivity to rotation since they result from an increased ratio between responses to rotation and translation. The reduction in the slope of the population response (Fig. 7f), which could theoretically be used by a downstream circuit to decode the axis of rotation, is proportional to the reduction in responses to rotation, and by extension 
a

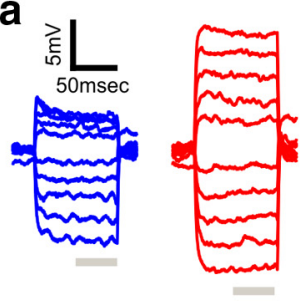

C

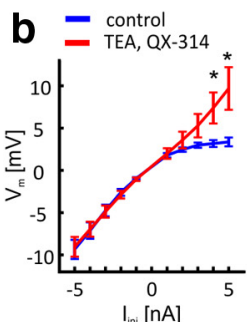

$\mathrm{I}_{\mathrm{inn}}[\mathrm{nA}]$ d

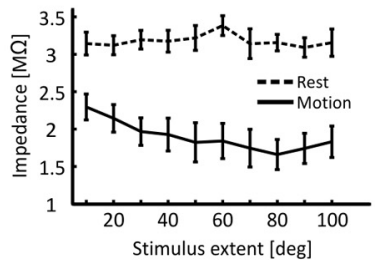

e

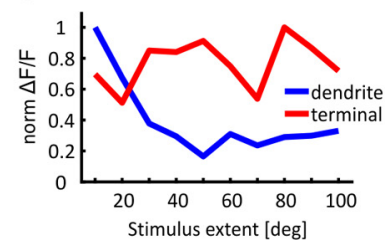

f

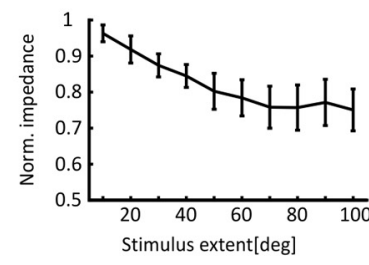

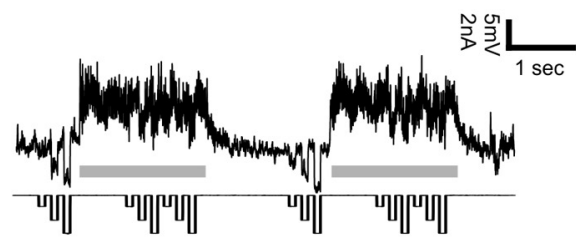

Figure 6. The effect of stimulus size on synaptic conductance in VS cells. $\boldsymbol{a}$, Membrane potential responses to current injections of -5 to $5 \mathrm{nA}$ in two VS3 cells. Left, Control (blue traces). Right, $100 \mathrm{~mm}$ TEA and $10 \mathrm{~mm}$ QX-314 in the tip solution. Gray bars - interval for averaging in $\boldsymbol{b}$. $\boldsymbol{b}$, Membrane potential averages in the control $(\mathrm{N}=5 \mathrm{cell} s)$ and the drug condition ( $N=6$ ). $\boldsymbol{c}$, Membrane potential responses of a VS cell to a $100^{\circ}$ drifting grating superimposed by current injections of various amplitudes. $\boldsymbol{d}$, Cell impedance for drifting gratings of varying lateral extent before and during motion, estimated from the current injections. $\boldsymbol{e}$, The same cell's dendritic and terminal calcium responses for the same gratings. $\boldsymbol{f}$, Average, normalized cell impedance for gratings of varying horizontal extent $(n=7)$.

smaller in size than the reduction in responses to translation in our model.

\section{Discussion}

In this work, we showed that the dendritic responses in VS and HS cells are inhibited by large-field motion in their preferred direction, but outside of the local dendritic excitatory receptive fields. This result is a distinct departure from the predominant view of LMD inputs, which are thought to be generated within independent visual "cartridges" and summed in a feedforward fashion on the LPTC dendrites. The inhibition is activated only by stimuli that extend in the direction of the gratings (i.e., perpendicular to the direction of motion), and not in the orthogonal direction. The similarities between end inhibition in VS and HS cells support the notion of a common underlying mechanism, which could be a reflection of the common mechanisms of motion detection in the horizontal and vertical systems in the lobula plate. In VS cells, the inhibition profile spreads laterally in a uniform fashion as far as our visual stimulus apparatus allowed. We showed evidence for a mechanism involving direct inhibitory inputs on the dendrites of VS cells. This form of lateral inhibition could be involved in enhancing selectivity to rotational flow fields versus translational flow fields.

Lateral suppression, a form of divisive normalization, is a common motif found in many sensory systems (Hartline et al., 1956; Roska et al., 2000; Olsen and Wilson, 2008; Wu et al., 2008; Carandini and Heeger, 2012), but its function and purpose are debated (Seriès et al., 2004; Oswald et al., 2006). In mammalian visual cortex, end-suppressed neurons in layers $2 / 3$ and 4 of primary visual cortex exhibit a strikingly similar form of surround suppression in the spatial domain (Hubel and Wiesel, 1965; Gilbert, 1977), and end suppression is also found in cortical areas responsive to large optic flow fields (Toyama et al., 1994). Although the mechanism is not clearly understood, it may involve an increase in inhibition driven by large-field layer 6 neurons (Bolz and Gilbert, 1986; Olsen et al., 2012); however, other authors suggest an underlying withdrawal of both excitation and inhibition (Ozeki et al., 2009). Interestingly, models of end suppression point toward its function in enhancing selectivity for direction of large-field motion, solving the aperture problem by incorporating information from outside the classical RF (Pack et al., 2003; Tsui et al., 2010). In essence, this is what our model shows, the aperture here being the small-field excitatory RF of VS cells, which do not contain sufficient information to discriminate between translation and rotation.

In the fly's lobula plate, lateral inhibition was found between VS1 and VS7/8, which are tuned to opposing axes of rotation (Haag and Borst, 2004, 2007). This also increases selectivity to rotation over translation (Borst and Weber, 2011), but the range of this inhibition in visual space is large $\left(\sim 100^{\circ}\right)$, without covering intermediate angles of visual space, and the site of inhibition is the axon terminal (Haag and Borst, 2007). Another form of lateral inhibition was described between laminar columns in the fly (Zettler and Järvilehto, 1972), but columnar neurons in this early processing stage are not direction selective and so are unlikely to be involved in the phenomenon we describe. A motionsensitive form of lateral inhibition was found in the locust, where it is thought to prevent adaptation to wide-field motion in the context of object detection (O'Shea and Rowell, 1975). Thus, the inhibitory effect we observed might be a reflection of phylogenetic similarities in the peripheral visual systems across various insect taxa (Buschbeck and Strausfeld, 1996).

Inhibition in the lobula plate is associated with two sources, the inhibitory subunits of the LMD outputs (Borst et al., 1995) and other inhibitory LPTCs. Of the $\sim 60$ LPTCs in each hemisphere, only 25 are well characterized; of these only centrifugal horizontal $(\mathrm{CH})$ cells have inhibitory dendritic output synapses, but they do not target VS or HS cells (Farrow et al., 2003; Haag and Borst, 2007) and so cannot explain the phenomenon we describe. However, we cannot rule out other, uncharacterized LPTCs as the source of inhibition. In our view, it is more likely that lateral inhibition utilizes existing LMD inhibitory input channels, without the need to postulate a second source of inhibition within the lobula plate.

VS and HS cells are both presynaptic in the axon terminal, whereas HS cells are also connected to other LPTCs via dendrodendritic gap junctions (Haag and Borst, 2002, 2007; Farrow et al., 2003). This connection to the inhibitory $\mathrm{CH}$ cells is thought to play a role in large-field inhibitory responses involved in the small-field selectivity of figure detection (FD) cells (Egelhaaf, 1985; Warzecha et al., 1993). FD cells are the only known exam- 
a
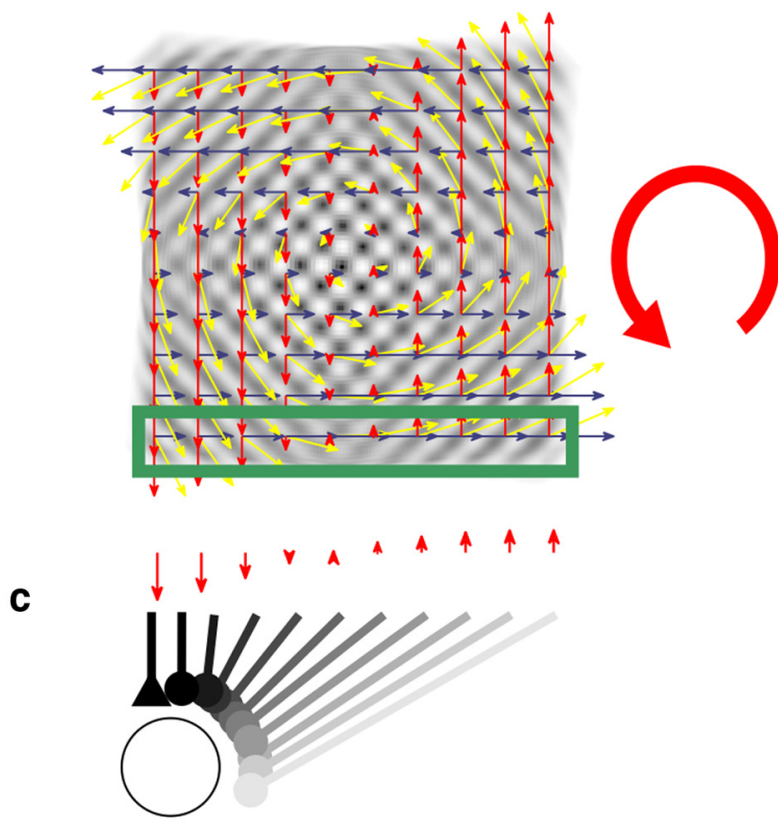

b

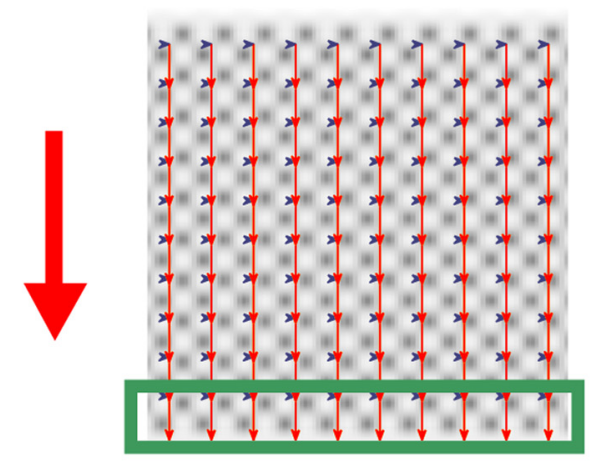

d
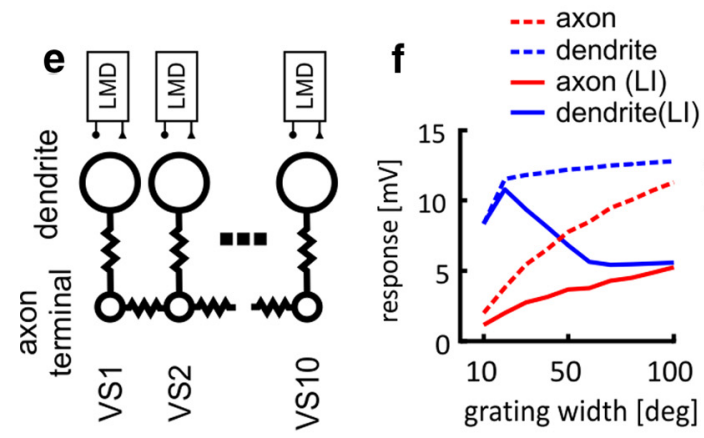

I

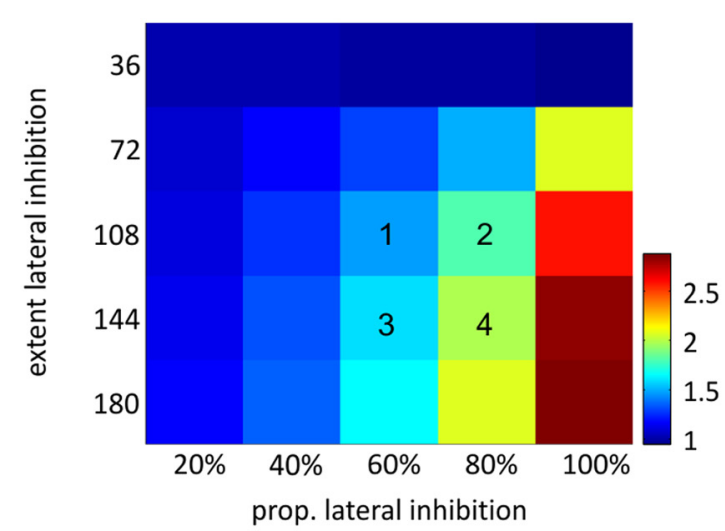

g

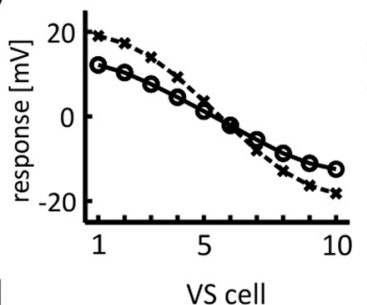

VS cell

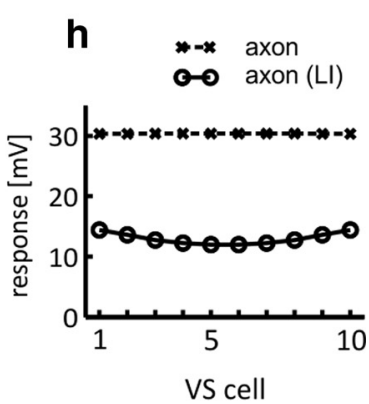

VS cell

j
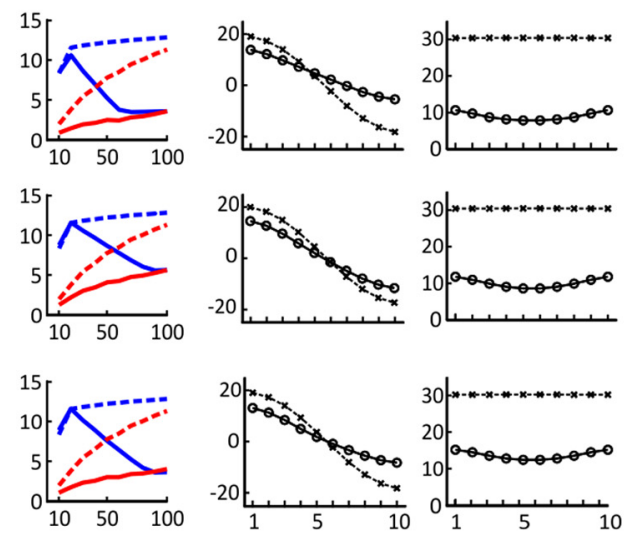

grating widt

[deg]

Figure 7. Lateral inhibition enhances selectivity to rotation in a VS network model. $\boldsymbol{a}$, Responses of a $100 \times 100$ array of Reichardt-type LMDs to rotation of an image constructed from a low-pass filtered checkerboard. Red arrows, Vertical LMDs; blue arrows, horizontal LMDs; yellow arrows, vector sum; averages over $10 \times 10$ pixel blocks. $\boldsymbol{b}$, Same as $\boldsymbol{a}$ for image translation. $\boldsymbol{c}$, Responses (red arrows) to the rotating image from a single row of the vertical LMD array (green box in $\boldsymbol{a}$ ), together with a diagram depicting the LI contributions from each LMD to a VS1 cell. Triangle, Excitatory; circle, inhibitory; darker shades represent larger amplitudes. $\boldsymbol{d}$, Same as $\boldsymbol{c}$ for the translating image. $\boldsymbol{e}$, Connectivity diagram for the VS network model. LMD represents an array of LMDs with inhibitory and excitatory outputs. Dendritic compartments (top) connected with axons to axon terminal compartments (bottom), which are connected in a chain-like fashion between themselves. $\boldsymbol{f}$ - $\boldsymbol{h}$, Responses of 1 of the 25 simplified VS network models to image motion, with and without LI. Model chosen for best fit with measured responses (LI extent, $108^{\circ} ; \mathrm{LI}$ proportion, $60 \%$ ). $\boldsymbol{f}$, Responses of a VS5 cell to drifting gratings of varying lateral extent for dendritic (blue) and axon terminal compartments (red), with (solid line) and without (broken line) LI. $\boldsymbol{g}$, Responses in the axon terminals of the VS population to the rotating checkerboard image, with (solid, circles) and without (broken, Xs) LI. $\boldsymbol{h}$, Same as $\boldsymbol{f f o r}$ the translating image. $\boldsymbol{i}$, Ratio of inhibition during translation to inhibition during rotation for all models in VS1, which is driven most strongly by this rotating image. Model 1 responses are shown in $\mathbf{e}-\boldsymbol{g}$, model $2-4$ responses in $\boldsymbol{g}$. $\boldsymbol{j}$, Three models presented as in $\boldsymbol{e}-\boldsymbol{g}$, corresponding to models $2-4$ in $\boldsymbol{h}$. 
ples of small-field selectivity in the lobula plate. However, their size selectivity is in the horizontal dimension, in contrast with size selectivity we found in HS cell dendrites, which is in the vertical dimension. We therefore do not consider an important role for small-field selectivity of HS dendrites in the generation of small-field selectivity in FD cells. Nevertheless, models of FD cell function require some form of distributed inhibition to achieve small-field selectivity (Hennig et al., 2008), similar to what we have found in the LI model, pointing out a general principle common to both systems.

The possibility of the existence of a similar, vertically sensitive small-field selective element in the lobula plate has been raised in another study of fly optomotor responses (Hausen and Wehrhahn, 1990), but such a cell in the lobula plate has not yet been found. Here we describe a mechanism that could easily be used by a downstream neuron to generate vertical, small-field selective optomotor responses.

We found a substantial discrepancy between the lateral extent of the suppression in VS cells measured using large-field gratings $\left(\sim 40^{\circ}\right)$ (Fig. $\left.3 a, b\right)$ and using laterally displaced gratings $\left(\geq 70^{\circ}\right)$ (Fig. $3 d$ ). This can be explained by the different sources of calcium influx into VS cells, a mixture of acetylcholine receptors (AChRs) and voltage-activated calcium channels (VACCs) (Egelhaaf and Borst, 1995; Borst and Single, 2000). Suppression by medium- and large-sized gratings (i.e., $>40^{\circ}$ ) could restrict dendritic excitation beneath the threshold of the VACCs, whereas additional inhibitory drive would not reduce the voltageindependent AChR response any further. This interpretation is also reflected in the reduction of impedance, which we measured up to a grating size of $60^{\circ}$ (Fig. 6). The absence of excitatory, AChR-mediated calcium influx for lateral stimuli is evidence that motion-dependent excitation in LPTC dendrites is not laterally distributed as its inhibitory counterpart.

The conclusions we draw rely significantly on a monotonic relationship between membrane potential and calcium responses in LPTCs during depolarizing stimulation. Voltage-clamp experiments demonstrated that VACC influx is linearly related to membrane potential in both the dendrite and the axon terminal within the regime of visual responses to preferred direction motion (Haag and Borst 2000). Although the slopes of these linear relations are different, they are all monotonic, allowing us to interpret a reduction in calcium influx as a reduction in membrane potential.

We found dendritic end inhibition in both VS and HS cells, suggesting a common mechanism in both axes of motion detection. Suppression magnitude differs between the two cell types ( $\sim 40 \%$ in VS cells, $\sim 25 \%$ in HS cells), which could result from the difference in the cells' structure. The narrow, dorsolateral VS cell dendrites are not driven by laterally positioned gratings, whereas the rounder HS cell dendrites can be excited by longitudinal gratings ( Dürr and Egelhaaf, 1999), by passive or active conduction, counteracting the effect of lateral inhibition.

Interestingly, the same gratings that inhibit a VS cell's dendrite cause a depolarization in the axon terminal, mediated by gapjunction coupling to neighboring VS cells (Haag and Borst, 2004; Farrow et al., 2005; Cuntz et al., 2007; Elyada et al., 2009). This is puzzling since the main output of VS cells is in the axon terminal. Spatial pooling of responses in VS cell axon terminals has an important role in smoothing the noisy local motion patterns that result from nonuniform visual input in naturalistic scenes (Cuntz et al., 2007; Elyada et al., 2009). This functionality in VS cells is supported by the electrotonic separation of synaptic LMD inputs in the dendrite from gap-junction connectivity in the axon ter- minal (Elyada et al., 2009). A possible explanation for this apparent conflict is that suppression by a spatially averaged signal is also a form of lateral averaging, different than simply having weaker gap-junction coupling to neighboring cells. Indeed, our modeling results show that the large-field integration in the axon terminals of VS cells that supports this smoothing can be maintained even when their dendrites are small field selective. Our model also suggests a possible function for dendritic end inhibition in enhancing selectivity to rotational flow fields, despite the "masking" of dendritic small-field selectivity in the cells' axon terminal.

Our previous work showed electrotonic separation of chemical synapses from gap junctions in VS cells, supporting a twotiered processing structure where the two sources of input do not interfere with each other's function (Elyada et al., 2009). Here we show that within a single cell, the seemingly conflicting sensory transformations of lateral inhibition and lateral coupling can be separated electrotonically to complement each other and to support their two distinct functionalities.

\section{References}

Bolz J, Gilbert CD (1986) Generation of end-inhibition in the visual cortex via interlaminar connections. Nature 320:362-365. CrossRef Medline

Borst A, Egelhaaf M (1992) In vivo imaging of calcium accumulation in fly interneurons as elicited by visual motion stimulation. Proc Natl Acad Sci U S A 89:4139-4143. CrossRef Medline

Borst A, Single S (2000) Local current spread in electrically compact neurons of the fly. Neurosci Lett 285:123-126. CrossRef Medline

Borst A, Weber F (2011) Neural action fields for optic flow based navigation: a simulation study of the fly lobula plate network. PLoS One 6:e16303. CrossRef Medline

Borst A, Egelhaaf M, Haag J (1995) Mechanisms of dendritic integration underlying gain control in fly motion-sensitive interneurons. J Comput Neurosci 2:5-18. CrossRef Medline

Borst A, Haag J, Reiff DF (2010) Fly motion vision. Annu Rev Neurosci 33:49-70. CrossRef Medline

Britten KH (2008) Mechanisms of self-motion perception. Annu Rev Neurosci 31:389-410. CrossRef Medline

Buschbeck EK, Strausfeld NJ (1996) Visual motion-detection circuits in flies: small-field retinotopic elements responding to motion are evolutionarily conserved across taxa. J Neurosci 16:4563-4578. Medline

Carandini M, Heeger DJ (2012) Normalization as a canonical neural computation. Nat Rev Neurosci 13:51-62. CrossRef Medline

Cuntz H, Haag J, Forstner F, Segev I, Borst A (2007) Robust coding of flowfield parameters by axo-axonal gap junctions between fly visual interneurons. Proc Natl Acad Sci U S A 104:10229-10233. CrossRef Medline

Duffy CJ, Wurtz RH (1991) Sensitivity of MST neurons to optic flow stimuli. I. A continuum of response selectivity to large-field stimuli. J Neurophysiol 65:1329-1345. Medline

Dürr V, EgelhaafM (1999) In vivo calcium accumulation in presynaptic and postsynaptic dendrites of visual interneurons. J Neurophysiol 82:33273338. Medline

Egelhaaf M (1985) On the neuronal basis of figure-ground discrimination by relative motion in the visual system of the fly. II. Figure-detection cells, a new class of visual interneurones. Biol Cybern 52:195-209.

Egelhaaf M, Borst A (1995) Calcium accumulation in visual interneurons of the fly: stimulus dependence and relationship to membrane potential. J Neurophysiol 73:2540-2552. Medline

Egelhaaf M, Warzecha AK (1999) Encoding of motion in real time by the fly visual system. Curr Opin Neurobiol 9:454-460. CrossRef Medline

Egelhaaf M, Borst A, Pilz B (1990) The role of GABA in detecting visual motion. Brain Res 509:156-160. CrossRef Medline

Elyada YM, Haag J, Borst A (2009) Different receptive fields in axons and dendrites underlie robust coding in motion-sensitive neurons. Nat Neurosci 12:327-332. CrossRef Medline

Farrow K, Haag J, Borst A (2003) Input organization of multifunctional motion-sensitive neurons in the blowfly. J Neurosci 23:9805-9811. Medline

Farrow K, Borst A, Haag J (2005) Sharing receptive fields with your neigh- 
bors: tuning the vertical system cells to wide field motion. J Neurosci 25:3985-3993. CrossRef Medline

Farrow K, Haag J, Borst A (2006) Nonlinear, binocular interactions underlying flow field selectivity of a motion-sensitive neuron. Nat Neurosci 9:1312-1320. CrossRef Medline

Franz MO, Krapp HG (2000) Wide-field, motion-sensitive neurons and matched filters for optic flow fields. Biol Cybern 83:185-197. CrossRef Medline

Geiger G, Nässel DR (1981) Visual orientation behaviour of flies after selective laser beam ablation of interneurones. Nature 293:398-399. CrossRef Medline

Gilbert CD (1977) Laminar differences in receptive field properties of cells in cat primary visual cortex. J Physiol 268:391-421. Medline

Haag J, Borst A (2000) Spatial distribution and characteristics of voltagegated calcium signals within visual interneurons. J Neurophysiol 83: 1039-1051. Medline

Haag J, Borst A (2002) Dendro-dendritic interactions between motionsensitive large-field neurons in the fly. J Neurosci 22:3227-3233. Medline

Haag J, Borst A (2003) Orientation tuning of motion-sensitive neurons shaped by vertical-horizontal network interactions. J Comp Physiol A Neuroethol Sens Neural Behav Physiol 189:363-370. CrossRef Medline

Haag J, Borst A (2004) Neural mechanism underlying complex receptive field properties of motion-sensitive interneurons. Nat Neurosci 7:628634. CrossRef Medline

Haag J, Borst A (2007) Reciprocal inhibitory connections within a neural network for rotational optic-flow processing. Front Neurosci 1:111-121. CrossRef Medline

Haag J, Theunissen F, Borst A (1997) The intrinsic electrophysiological characteristics of fly lobula plate tangential cells: II. Active membrane properties. J Comput Neurosci 4:349-369. CrossRef Medline

Haag J, Wertz A, Borst A (2010) Central gating of fly optomotor response. Proc Natl Acad Sci U S A 107:20104-20109. CrossRef Medline

Hartline HK, Wagner HG, Ratliff F (1956) Inhibition in the eye of Limulus. J Gen Physiol 39:651-673. CrossRef Medline

Hausen K, Egelhaaf M (1989) Neural mechanisms of visual course control in insects. In: Facets of vision (Stavenga D, Hardie R, eds), pp 391-424. Berlin, Heidelberg, New York: Springer-Verlag.

Hausen K, Wehrhahn C (1990) Neural circuits mediating visual flight control in flies. II. Separation of two control systems by microsurgical brain lesions. J Neurosci 10:351-360. Medline

Heisenberg M, Wonneberger R, Wolf R (1978) Optomotor blind H31 a Drosophila mutant of the lobula plate giant neurons. J Comp Physiol 124:287-296. CrossRef

Hennig P, Möller R, Egelhaaf M (2008) Distributed dendritic processing facilitates object detection: a computational analysis on the visual system of the fly. PLoS One 3:e3092. CrossRef Medline

Hubel DH, Wiesel TN (1965) Receptive fields and functional architecture in two nonstriate visual areas (18 and 19) of the cat. J Neurophysiol 28:229289. Medline

Krapp HG, Hengstenberg R (1996) Estimation of self-motion by optic flow processing in single visual interneurons. Nature 384:463-466. CrossRef Medline
Krapp HG, Hengstenberg R, Egelhaaf M (2001) Binocular contributions to optic flow processing in the fly visual system. J Neurophysiol 85:724-734. Medline

Olsen SR, Wilson RI (2008) Lateral presynaptic inhibition mediates gain control in an olfactory circuit. Nature 452:956-960. CrossRef Medline

Olsen SR, Bortone DS, Adesnik H, Scanziani M (2012) Gain control by layer six in cortical circuits of vision. Nature 483:47-52. CrossRef Medline

O'Shea M, Rowell CH (1975) Protection from habituation by lateral inhibition. Nature 254:53-55. CrossRef Medline

Oswald AM, Schiff ML, Reyes AD (2006) Synaptic mechanisms underlying auditory processing. Curr Opin Neurobiol 16:371-376. CrossRef Medline

Ozeki H, Finn IM, Schaffer ES, Miller KD, Ferster D (2009) Inhibitory stabilization of the cortical network underlies visual surround suppression. Neuron 62:578-592. CrossRef Medline

Pack CC, Livingstone MS, Duffy KR, Born RT (2003) End-stopping and the aperture problem: two-dimensional motion signals in macaque V1. Neuron 39:671-680. CrossRef Medline

Roska B, Nemeth E, Orzo L, Werblin FS (2000) Three levels of lateral inhibition: a space-time study of the retina of the tiger salamander. J Neurosci 20:1941-1951. Medline

Seriès P, Latham PE, Pouget A (2004) Tuning curve sharpening for orientation selectivity: coding efficiency and the impact of correlations. Nat Neurosci 7:1129-1135. CrossRef Medline

Single S, Borst A (1998) Dendritic integration and its role in computing image velocity. Science 281:1848-1850. CrossRef Medline

Tanaka K, Fukada Y, Saito HA (1989) Underlying mechanisms of the response specificity of expansion/contraction and rotation cells in the dorsal part of the medial superior temporal area of the macaque monkey. J Neurophysiol 62:642-656. Medline

Toyama K, Mizobe K, Akase E, Kaihara T (1994) Neuronal responsiveness in areas 19 and 21a, and the posteromedial lateral suprasylvian cortex of the cat. Exp Brain Res 99:289-301. Medline

Tsui JM, Hunter JN, Born RT, Pack CC (2010) The role of V1 surround suppression in MT motion integration. J Neurophysiol 103:3123-3138. CrossRef Medline

Warzecha AK, Egelhaaf M, Borst A (1993) Neural circuit tuning fly visual interneurons to motion of small objects. I. Dissection of the circuit by pharmacological and photoinactivation techniques. J Neurophysiol 69: 329-339. Medline

Wertz A, Gaub B, Plett J, Haag J, Borst A (2009) Robust coding of egomotion in descending neurons of the fly. J Neurosci 29:14993-15000. CrossRef Medline

Wu GK, Arbuckle R, Liu BH, Tao HW, Zhang LI (2008) Lateral sharpening of cortical frequency tuning by approximately balanced inhibition. Neuron 58:132-143. CrossRef Medline

Wylie DR, Bischof WF, Frost BJ (1998) Common reference frame for neural coding of translational and rotational optic flow. Nature 392:278-282. CrossRef Medline

Zettler F, Järvilehto M (1972) Lateral inhibition in an insect eye. Z Vgl Physiol 76:233. CrossRef 